\title{
Biology Research Progress on Trichomonas vaginalis in China
}

\author{
Haiguang Cai, Qi Liao, Dingli Xu, Tuergong Jiang, Xianqun Rao, Yanting Zhang, Ding Chen, \\ Rui Chen, Yiyuan Zhang, Huihui Xie, Jianfa Liu*
}

Medical School of Ningbo University, Ningbo, China

Email: ${ }^{*}$ liujianfa@nbu.edu.cn

Received 19 April 2015; accepted 10 May 2015; published 19 May 2015

Copyright $@ 2015$ by authors and OALib.

This work is licensed under the Creative Commons Attribution International License (CC BY). http://creativecommons.org/licenses/by/4.0/

(c) (i) Open Access

\section{Abstract}

Trichomonas vaginalis is one of the most common human sexually transmitted disease pathogen that colonizes the urogenital mucosa. Based on the research status of Trichomonas vaginalis infection in China, the training methods, pathogenic mechanisms and treatment on this parasite has been reviewed in this paper.

Keywords

Trichomonas Vaginalis, Biology, Research Progress

Subject Areas: Infectious Diseases, Public Health

\section{Introduction}

Trichomonas vaginalis is a common secrete genital tract parasite, men and women are infected, by direct or indirect contact with the transition of the host and spread. The harm is that a parasite in the vagina or other urogenital organs causes vaginitis, urethritis or infertility. There are reports the Trichomonas vaginalis infections could also increase the chances of HIV infection [1] and other chances of infections of other sexually transmitted diseases [2]. Trichomonas vaginalis infection is more common in recent years, for Trichomonas vaginalis in all aspects of biological research is taken seriously increasingly, the study on the pathogenic mechanism is gradually growing, the drugs against Trichomonas vaginalis is also gradually developing, so in this paper, biology research progress for Trichomonas vaginalis.

\section{The Present Situation of Trichomonas Vaginalis Infections}

The World Health Organization statistics showed that more than 200 million or 300 - 400 million people world-

*Corresponding author.

Financially Supported by Natural Scientific Foundation of Ningbo City (2010A610036) and Subject Programme Foundation of Ningbo University (XKL14D2105).

How to cite this paper: Cai, H.G., Liao, Q., Xu, D.L., Jiang, T., Rao, X.Q., Zhang, Y.T., Chen, D., Chen, R., Zhang, Y.Y., Xie, H.H. and Liu, J.F. (2015) Biology Research Progress on Trichomonas vaginalis in China. Open Access Library Journal, 2: e1512. http://dx.doi.org/10.4236/oalib.1101512 
wide are infected with Trichomonas vaginalis each year. In the United States, there are different infection rates in different people in the different regions. Our country's Trichomonas vaginalis infection rate was higher. Our country in recent years have reported hospital department of gynaecology Trichomonas vaginalis infection rate is significantly higher than the average global female patient infection rate (3.1\%), and investigation of the prevalence of this disease is lower than the previous survey. This also reminds us that we should pay attention to the infection situation in order to prevent its spread, and pay attention to different regions and population infection, especially some hospital epidemic high-risk areas. As well as Trichomonas vaginalis infection does not have seasonal differences. It also accords with the general law of its epidemiology. At the same time the reported literature indicates that Trichomonas vaginalis infection with the infection of sexually transmitted diseases also has close relationship. In the process of globalization, our country has joined the trend as a participant, it also brings us some new changes, such as sex crime, drug use and sexual intercourse, the spread of these behaviors greatly increased the infection of sexually transmitted diseases, this also directly or indirectly increases the infection of Trichomonas vaginalis. So we should study the infection mechanism and pathogenic mechanism more in-depthly and reduce the infection rate.

\section{On Trichomonas Vaginalis in Vitro}

In recent years the onset of Trichomonas vaginalis is rising to strengthen the research on disease of Trichomonas vaginalis. Scientists used RPMI1640 culture for cultivation of Trichomonas vaginalis in vitro [3], the medium include liver immersion medium suitable for Trichomonas vaginalis survival, drug tests and other experimental research of the project.

\section{The Trichomonas Vaginalis Virus Research}

Trichomonas vaginalis virus include 7 kinds of protozoon virus, open loop, linear dsRNA, The viral genome sequencing and genome size presents diversity and polymorphism. cDNA clone of the four kinds of virus (TVVT1, TVV-type I, TVV-type II, TVV-type III) [4] between different length and homology is also relatively low, but it has the ability of encoding protein. Its action also failed to clear. Virus replication method has been reported, which needs us to research. But we have learned that the presence of Trichomonas vaginalis virus of insect body grow and divide without significant effect, but with the pathogenicity of the insect body, immunogenicity and drug resistance have certain relations. The presence of the virus can also affect the insect body protein expression. So we should increase the research of virus, and from this area we need to find the pathogenesis of trichomoniasis.

\section{Research on the Treatment of Trichomonas Vaginalis}

\subsection{Chinese Bulbul against Trichomonas Vaginalis Pharmacological Effects}

In recent years, studies have shown that the Chinese bulbul has obvious killing activity on Trichomonas vaginalis. Experiments show that after using the drug, Trichomonas vaginalis obviously changed in ultrastructure. After drug action, the patient's body appeared a large number of vacuoles. For a long time, a variety of organelles involved, breeding and protein synthesis of trichomonad are also lethally damaged. This makes Chinese bulbul's resistance to the pharmacological actions of trichomonas vaginalis possible [5].

\subsection{The Total Base of Trichomonas Vaginalis Drugs of Sophora Alopecuroides}

Total alkaloid of sophora alopecuroides isolated from sophora alopecuroides, according to previous reports on Escherichia coli, have obvious inhibitory effects on parasites, and it also has therapeutic effect on other diseases. In China, there was a report that the drug had a strong role Trichomonas vaginalis [6].

\subsection{The Pharmacological Actions of Chinese Medicine Chinese Prickly Ash against Trichomonas Vaginalis}

In vitro experimental study, it has been showed that Chinese prickly ash decoction has obvious inhibition on Trichomonas vaginalis. When the concentration of Chinese prickly ash decoction is more than $6.25 \%$, the activity against Trichomonas vaginalis could reach one hundred percent.. So the properly prepared Chinese prickly 
ash can be used for treatment of trichomonas vaginitis [7].

\subsection{Compound Chinese Herbal Medicine against Trichomonas Vaginalis Pharmacological Effects}

Compound Chinese medicine for a variety of traditional Chinese medicine, is more complicated in pharmacology. Scholars in China have summarized many traditional Chinese medicines against Trichomonas vaginalis, which appeared insecticidal itching, diminish inflammation. After 30 minutes, the vast majority of the nuclear membrane damage, karyoplasm disappear or loose [8]. This shows that JieErYin has strong killing effect on Trichomonas vaginalis. We can learn from it to prevent the breeding and spread of trichomonad.

\subsection{Double Hydrogen Green Pole Element Resistance to Pharmacological Effects of Trichomonas Vaginalis}

Double hydrogen green pole element is one of the derivative of the green pole element, is an extensively drug that resists parasites and has little adverse reaction. By in vitro experiments, the drug has very strong insecticidal effect on Trichomonas vaginalis [8]-[10].

\section{The Role of Lactobacillus on Trichomonas Vaginalis}

Lactobacillus is the dominant fungi living in healthy women vaginala, microbial balance plays an important role in maintaining vaginal environment. Under normal circumstances, healthy women's vaginal environment is acidic microenvironment produced by lactic acid bacteria [11] that could inhibit the parasite's growth and reproduction [12]. The experimental results show that $0.5 \%$ of lactic acid concentrations, the Trichomonas vaginalis all died after 2 hours action [12]. Lactic acid bacteria concentration in a certain range has strong inhibitory effect. Through the experiment, we can make preventive measures from the aspects of maintaining lactobacillus concentration of Trichomonas vaginalis infection. This reduces the infection of Trichomonas vaginalis.

\section{Molecular Pathogenesis Mechanism of Trichomonas Vaginalis}

Micro-environment is the foundation of Trichomonas vaginalis erosion target cell disease, that is a necessary environment for trichomonad disease and trichomonad itself. Foreign scholars reported trichomonad existed adhesion factor, and confirmed that adhesion factor was the indispensable material in the pathogenic mechanism of Trichomonas vaginalis. Radioactive marker and electrophoresis confirmed Trichomonas vaginalis adhesion factor is composed of five kinds of protein, respectively AP120, AP65, AP51, AP33, AP23 [13]. Adhesion molecules is the combination of mediated trichomonad and target cells, when trichomonad contact with the vaginal epithelial cells, trichomonad can the protein synthesis. The second mode of trichomonad contact with target cells of trichomonad and vaginal epithelium extracellular matrix and fiber adhesion proteins in layer adhesion proteins are linked together. After contacting trichomonad signal system (mainly for the G protein), trichomonad actin began to increase new translation expression and distribution of the cytoskeleton changes, which benefited the mobile of trichomonad [14].

\section{Trichomonas Vaginalis Research of Recombinant Proteins}

Reports proved that Trichomonas vaginalis could have five kinds of adhesions in epithelial cells of genitourinary tract, including surface adhesion protein AP33 which is coding MR33000 protein. The protein of leader peptide has been confirmed as Trichomonas vaginalis AP33 protein adhesion molecules. The determination of the protein AP33 is very important. The experiments also confirm AP33 protein has strong immunogenicity, and can stimulate the body to produce and protein, which have the effect on inhibiting trichomonad breeding. Some scholars in China had used DNA separation and construction of prokaryotic expression system, and western blot immune double diffusion test and clinical separation of Trichomonas vaginalis worm strains to investigate the mechanisms of Trichomonas vaginalis infections [15].

The rate of Trichomonas vaginitis caused by trichomonas vaginalis infection is higher and higher, which seriously affects our health. So we should strengthen the research on diagnosis, prevention and treatment of this parasite. 


\section{References}

[1] Ma, J.H., Gao, J.L. and Yu, D. (1999) Trichomonas vaginalis in Parasitic in Patients with Sexually Transmitted Diseases. Chinese Journal of Prevention and Control of Parasitic Diseases, 12, $28-42$.

[2] Yang, J., Yao, F.R. and Xu, Z. (2004) Trichomonas vaginalis in Two Kinds of Culture Medium in the Growth and Morphological Observation. Chinese Journal of Prevention and Control of Parasitic Diseases, 17, 14-19.

[3] Zhao, Y.P.. Zhang, X.C., Liu X.F. and Tao, G.P. (2008) Trichomonas vaginalis Virus Research Progress. Chinese Journal of Pathogenic Biology, 3, 28-32.

[4] Yan, Y. and Gao, X.Z. (2006) Chinese Traditional Medicine against Trichomonas vaginalis in Vitro Projection with Electron Microscope. Chinese Journal of Pathogenic Biology, 1, 32-38.

[5] Zhao, R., Li, Y.B. and Huang, L. (2004) Total Alkaloid of Sophora alopecuroides and Four Kinds of Biological Single Alkali Tag of Trichomonas vaginalis in Vitro Experiment Study. Traditional Chinese Medicine in Sichuan, 22, 12-16.

[6] Zhang, D.X. (2003) The Role of Traditional Chinese Medicines against Trichomonas vaginalis in Vitro Study. Journal of Lanzhou Medical College, 29, 23-28.

[7] Mao, Y. (2006) The Pharmacological Effects of Chinese Herbal Medicine against Trichomonas vaginalis Research Progress. Parasites and Infectious Diseases, 4, 28-32.

[8] Shang, Z.H., Zhou, X.O. and Gao, X.Z. (2007) Double Hydrogen Green Pole Element with Electron Microscope to Observe the Role of in Vitro Cultivation of Trichomonas vaginalis. Chinese Journal of Parasitology and Parasitic Disease, 25, 38-41.

[9] Xu, J.B. and Gao, X.Z. (2004) Double Hydrogen Green Pole Element of Trichomonas vaginalis Microfilament Effect Observation. Chinese journal of Prevention and Control of Parasitic Diseases, 17, 48-52.

[10] Gu, T.T., Qiao, J.Y., Yang, J.H., et al. (2004) Lactic Acid Bacteria Produces the Effect of the Microenvironment of Trichomonas vaginalis Mice Model. Journal of Tropical Medicine, 4, 12-16.

[11] Shang, Z.H., Xu, J.B., Gao, M.J., et al. (2011) The Research Progress of Trichomonas vaginalis Molecular Pathogenic Mechanism. Chinese Journal of Parasitology and Parasitic Diseases, 29, 28-33.

[12] Chen, Q., Qiao, J.J., Lu, C.H., et al. (2004) Lactobacillus Micro Environment Caused by the Impact on Trichomonas vaginalis. Chinese Journal of Microecology, 16, 28-32.

[13] Li, S.G., Ziguang Jiang, Z.G., Fan, H.W. and Jin, H. (2008) Investigating the Situation of Trichomonas vaginalis Infection in Patients with Gynecological Clinic Analysis. Maternal and Child Health Care in China, 23, 36-40.

[14] Liang, S.H., Huang, H.C., Pan, C.W., et al. (2006) Preparation of Recombinant Protein AP33 of Trichomonas vaginalis, Identification and Preliminary Application. Chinese Journal of Parasitology and Parasitic Diseases, 24, 42-46.

[15] Alderete, J.F. and Garza, G.E. (1988) Identification and the Properties of Trichomonas vaginalis Proteins Involved in Cytoadherence. Infection and Immunity, 56, 28-33. 